\title{
Effects of different patterns of reinforcement on performance under massed or spaced extinction*
}

\author{
N. J. MACKINTOSH and LYDIA LITTLE \\ Dalhousie University, Halifax, N.S., Canada
}

Two groups of rats received two trials a day in a runway in the sequence RN or NR, and were then extinguished either in a single session or at a rate of one trial per day. With massed trials, Group NR extinguished more slowly; with spaced trials, Group RN tended to extinguish more slowly. These results are consistent with Capaldi's sequential theory. A second experiment, however, showed that the difference in spaced extinction was not due to differences in between-day transitions (as Capaldi's analysis might imply), but depended on whether or not Trial 1 of each day in acquisition was reinforced.

Grosslight \& Radlow (1956, 1957), studying rats in a discrimination learning situation, found that when the proportion of rewarded $(\mathrm{R})$ to nonrewarded $(\mathrm{N})$ trials in acquisition was held constant, resistance to extinction depended on the order in which $\mathbf{R}$ and $\mathrm{N}$ trials were scheduled each day. A schedule in which $\mathrm{N}$ trials occurred early in each day resulted in greater resistance to extinction than one in which all $\mathrm{N}$ trials occurred at the end of each day. Spivey \& Hess (1968) reduced the experimental design to its apparent essentials by comparing resistance to extinction in a runway following acquisition training in which only two trials were given each day-either in the order NR or in the order RN. A group trained with the NR sequence was more resistant to extinction than one trained with the RN sequence. Analogous results have been reported by Leonard (1969) in a varied magnitude-of-reward experiment: $A$ group receiving two trials a day with a small reward on the first trial and a large reward on the second (SL) was more resistant to extinction than one receiving the opposite sequence (LS).

These results are satisfactorily explained by Capaldi's sequential theory, which states that resistance to extinction is determined by the extent to which the instrumental response has been conditioned to stimuli similar to the aftereffects of $\mathbf{N}$ trials experienced in extinction (Capaldi, 1967). When a series of extinction trials is given in a single session, Group NR, which has been rewarded for running immediately following an $\mathrm{N}$ trial, will continue to perform the instrumental response longer than Group RN, which was not rewarded in acquisition immediately after $\mathrm{N}$ trials. Leonard's results can be explained by the reasonable assumptions that different magnitudes of reward vary along a

*This research was supported by Grant APA 259 from the National Research Council of Canada. continuum, one of whose endpoints is defined by nonreward, and that small rewards lie closer to nonreward than do large rewards. However, as Leonard, Albin, \& Lebowitz (1969) have pointed out, the predictions from Capaldi's theory only apply if extinction trials are given in a single session. If extinction trials were given at a rate of one per day, the relevant transitions would be those occurring between days during acquisition, and, according to a strict reading of Capaldi's theory, Group RN, which is rewarded in acquisition $24 \mathrm{~h}$ after an $\mathrm{N}$ trial, will be more resistant to extinction than Group NR. Using LS and SL sequences, Leonard et al confirmed this rather striking prediction: They found that when extinction trials were massed, Group SL extinguished more slowly than Group LS, but when extinction trials were given one per day, the ordering of the groups was reversed. Experiment 1 tested the same prediction using NR and RN sequences.

\section{EXPERIMENT 1} Method

The Ss were 32 male hooded rats, about 100 days old, maintained at $85 \%$ of their ad lib weights. The apparatus was a grey runway, $32 \times 6 \times 6$ in., with a hinged Perspex roof. Perspex guillotine doors divided off the first 8 in. and the final 12 in. as start- and goalbox. Opening the startbox door started a .10-sec clock which stopped when $S$ interrupted a photobeam 3 in. inside the goalbox.

The Ss were placed on deprivation 10 days before the start of training, given one 10-min period of exploration in the runway (without food), and on the following day placed three times in the goalbox where they were left until they ate a single 190-mg Noyes pellet. During acquisition, all $S s$ received two trials per day, with a 30-sec intertrial interval, for 15 days. Half the Ss (Group NR) were rewarded on Trial 2 each day, the remainder (Group RN) on Trial 1. On N trials, Ss were detained in the goalbox for
$30 \mathrm{sec}$; on $\mathrm{R}$ trials they received one 190-mg Noyes pellet.

All Ss received 20 extinction trials. Half of each group (NR-massed and RN-massed) received all extinction trials, with a 30 -sec intertrial interval, on the day following the end of acquisition; the remaining Ss (NR-spaced and RN-spaced) received one extinction trial per day. On all trials, Ss were detained in the goalbox for $30 \mathrm{sec}$.

Results

By the end of acquisition, the two groups were beginning to learn their respective patterns of $\mathbf{R}$ and $\mathrm{N}$ trials, and an analysis of variance on Day 15 speeds revealed a significant interaction between groups and speeds on Trial 1 and Trial 2 $(F=4.74, \quad d f=1 / 30, \quad p<.05)$. This patterning, however, had only just appeared: On Days 12, 13, and 14 the interaction did not approach significance $(\mathrm{F}<1)$. Furthermore, an analysis of variance performed on performance on Trial 1 of extinction revealed no significant difference between groups ( $F=1.64$, $\mathrm{p}>.10$ ). Running speeds in extinction, averaged over five-trial blocks, are shown in Fig. 1. An analysis of variance performed on these scores revealed a significant effect of trial blocks $(F=44.42, d f=3 / 84$, $\mathrm{p}<.001$ ), and a significant interaction between pattern of reinforcement ( $\mathrm{RN}$ vs NR) and distribution of extinction trials $(F=6.97, \quad d f=1 / 28, \quad p<.025)$. When extinction trials were massed, Group NR ran more rapidly; when extinction trials were spaced, Group RN ran more rapidly. Separate analyses revealed that the group difference was significant under massed conditions $(F=5.14, \mathrm{df}=1 / 14, p<.05)$, but was not significant under spaced conditions $(F=2.16, p>.10)$.

These results are very similar to those reported by Leonard et al comparing LS and SL schedules. In both cases, the interaction between groups and distribution of extinction trials was

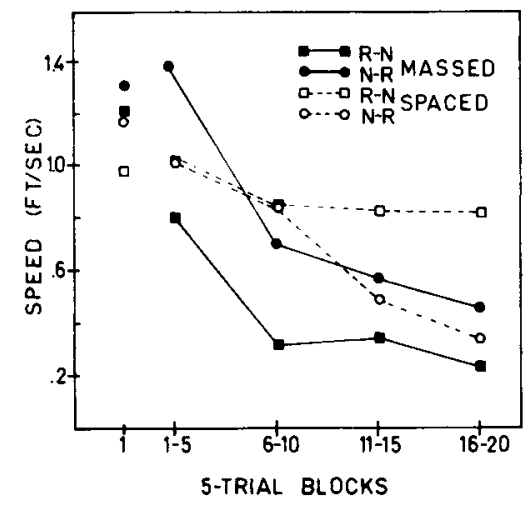

Fig. 1. Experiment 1. Mean speed for each group on Trial 1 and over 5-trial blocks in extinction. 


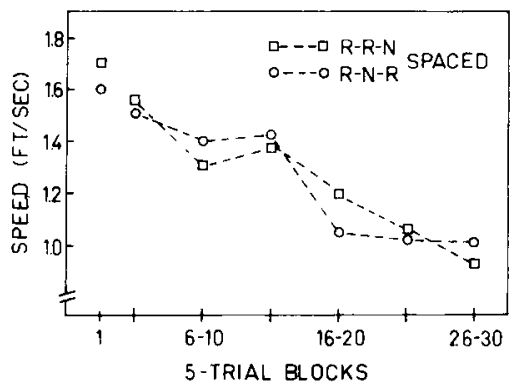

Fig. 2. Experiment 2. Mean speed for each group on Trial 1 and over 5-trial blocks in extinction.

significant, and separate analyses revealed a significant difference between groups in massed extinction, with a nonsignificant difference in the opposite direction under spaced extinction. This consistency suggests that, although small, the difference between groups under spaced extinction may well be a real one. If it is, although providing nice support for an aftereffects analysis, the result is surprising, for it implies that Ss are able to remember over a 24-h interval whether their last trial was $\mathrm{N}$ or $\mathrm{R}$. While Capaldi has usually assumed that the memory for an $\mathbf{N}$ or $\mathbf{R}$ trial persists intact until replaced, the evidence for this assumption is not particularly strong: If trained on an alternating RNRN sequence at a rate of one trial per day, rats do not usually show the appropriate pattern of running speeds they display when trials are massed (Surridge \& Amsel, 1966, 1968; Amsel, Hug, \& Surridge, 1969).

In fact, even though the difference between Groups NR and RN under spaced extinction was not significant, there is another possible explanation for it. The two groups differ not only in whether they were rewarded on the last trial of each day (the important factor according to an aftereffects analysis), but also in whether they were rewarded on the first trial of each day. It is reasonable to suppose that Trial 1 of each day is a distinctive event; when Ss received spaced extinction, each extinction trial is Trial 1 of the day; $S$ s in Group RN have been rewarded for running on Trial 1 and should therefore be more resistant to extinction than $\mathrm{Ss}$ in Group NR who have not been rewarded for running on Trial 1. This analysis leads to a simple prediction: if all $S s$ receive a rewarded trial at the beginning of each day in acquisition, it will make no difference to spaced trial extinction performance whether the last trial of the day is $N$ or $R$. In Experiment 2, therefore, two groups were given three daily acquisition trials, RRN or RNR, and then extinguished at a rate of one trial per day.

\section{EXPERIMENT 2 \\ Method}

The Ss were 16 male hooded rats from the same stock as those used in Experiment 1. The apparatus and, with two exceptions, the procedure were exactly the same as in Experiment 1. The first difference was that Ss received three trials per day in acquisition-either RRN or NRR; the second difference was that both groups received 30 spaced extinction trials. Results

Running speeds in extinction are shown in Fig. 2. There was no significant difference between the two groups either on Trial 1 of extinction or at any later time. An analysis of variance revealed a significant effect of five-trial blocks $(F=9.71$, df $=5 / 70, p<.001)$, but $F<1$ both for the group effect and the interaction between groups and blocks of trials.

These results suggest that the spaced extinction results of Experiment 1 and those reported by Leonard et al were not due to the type of between-day transition experienced in acquisiton, but to the conditions of reinforcement on Trial 1. The implication is that in Experiment 2 there was no difference between groups in the nature of the aftereffect conditioned on Trial 1 of each day, which in turn implies that Ss were unable to remember from one day to the next which event had occurred last. This is entirely consistent with the weight of the evidence on patterning effects at long intertrial intervals. It should not, however, be construed as proof that an aftereffects theory is inapplicable at long intertrial intervals: The fact that Ss cannot remember the order of two events over a long interval does not imply complete loss of memory for those events (Mackintosh, in press).

REFERENCES

AMSEL, A., HUG, J. J., \& SURRIDGE, C. T. Subject-to-subject trial sequence, odor trails, and patterning at 24-h ITI. Psychonomic Science, 1969, 15, 119-120.

CAPALDI, E. J. A sequential hypothesis of instrumental learning. In $\mathrm{K}$. W. Spence and $J$. T. Spence (Eds.), The psychology of learning and motivation: Advances in research and theory. Vol. 1. New York: Academic Press, 1967, Pp. 67-156.

GROSSLIGHT, J. H., \& RADLOW, R. Patterning effect of the nonreinforcement-reinforcement sequence in a discrimination situation. Journal of Comparative \& Physiological Psychology, $1956,49,542-546$.

GROSSLIGHT, J. H., \& RADLOW, R. Patterning effect of nonreinforcement-reinforcement sequence involving a single nonreinforced trial. Joumal of Comparative \& Physiological Psychology, 1957, 50, 23-25.

LEONARD, D. W. Amount and sequence of reward in partial and continuous reinforcement. Journal of Comparative \& Physiological Psychology, 1969, 67, 204-211.

LEONARD, D. W., ALBIN, R., \& LEBOWITZ, $M$. Performance under massed or spaced extinction following different sequences of varied reward training. Psychonomic Science, 1969, 16, 130-132.

MACKINTOSH, N. J. Distribution of trials and the partial reinforcement effect. In preparation.

SPIVEY, J. E., \& HESS, D. T. Effect of partial reinforcement trial sequences on extinction performance. Psychonomic Science, 1968, 10, 375-376.

SURRIDGE, C. T., \& AMSEL, A. Acquisition and extinction under single alternation and random partial-reinforcement conditions with a 24-hour intertrial interval. Journal of Experimental Psychology, 1966, 72, 361-368.

SURRIDGE, C. T., \& AMSEL, A. Confinement duration on rewarded and nonrewarded trials and patterning at 24-hour ITI. Psychonomic Science, 1968, 10, 107-108. 